\title{
Gene tests for brain injury still far from the football field
}

Last month, doctors released the autopsy results of former Chicago Bears star Dave Duerson, confirming speculation that the professional football player had suffered from chronic traumatic encephalopathy (CTE), a form of dementia thought to be caused by years of head injuries sustained on the field. Did he carry a genetic signature that predisposed him to the disease? For almost two decades researchers have hunted-some would say in vain-for genes that might make people more susceptible to dementia from traumatic brain injuries. In February, just days after Duerson committed suicide by a shot to the stomach, researchers published another paper that touched on the role genes might have in CTE, but the connection remains controversial.

"If we had a genetic marker that was really associated with a high prevalence of CTE, people with genetic risk factors might learn about them and choose another sport," says Robert Cantu, co-director of the Center for the Study of Traumatic Encephalopathy at the Boston University School of Medicine.

CTE has similarities to Alzheimer's diseaseincluding its symptoms and its pathology of tangles and plaques in the brain. That's why, soon after the early-1990s discovery that individuals carrying the $\varepsilon 4$ variant of apolipoprotein $\mathrm{E}$ (encoded by APOE) have an increased risk of Alzheimer's disease, researchers began probing that gene's association with brain injury-induced dementia. A handful of studies subsequently reported that among people who had suffered traumatic brain injuries, those who were $\varepsilon 4$ carriers did not tend to recover as well as individuals with other APOE alleles. Mouse experiments also supported this link.

In one of the first studies to explicitly investigate athletes' ApoE status, Barry Jordan, now the director of the brain injury program at the Burke Rehabilitation Hospital in White Plains, New York, assessed cognitive, behavioral and motor symptoms in a group of 30 boxers using a clinical scale of brain injury; those who scored higher tended to have the $\varepsilon 4$ variant. In a subgroup who had had the most exposure to the sport, the mean brain injury score for athletes with $\varepsilon 4$ was double that of those with $\varepsilon 3$ and ع2 (J. Am. Med. Assoc. 278,136-140, 1997). A later study in football players conducted by the same group found a link between the allele and deteriorating memory, attention and reaction time (Neurosurgery 47, 651-657, 2000). More recently, Cantu coauthored an analysis of all CTE cases verified via autopsy in the literature; results from the ten of those that had been genotyped for ApoE status also suggested

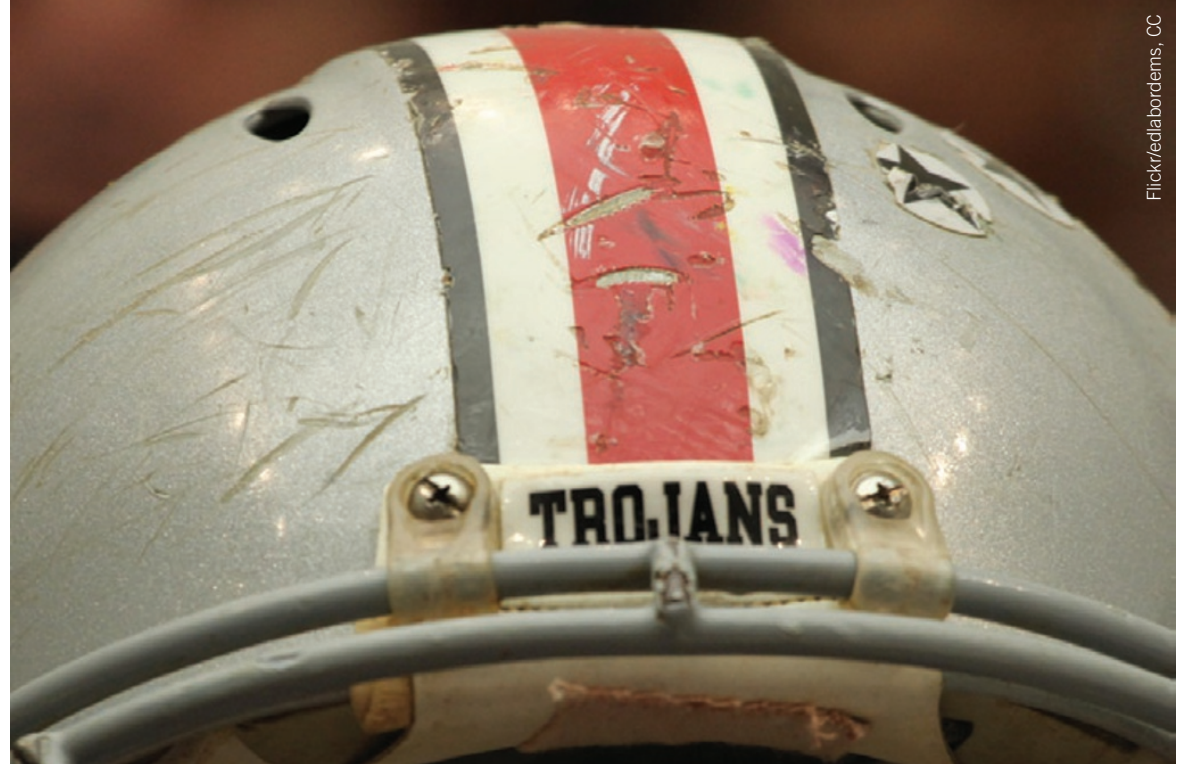

Broken dreams: The helmet of Owen Thomas, a college footballer with CTE who committed suicide.

poorer outcomes for $\varepsilon 4$ carriers (Neuropathol. Exp. Neurol. 68, 709-735, 2009).

Alzheimer's and CTE might be linked via a multitude of mechanisms mediated by ApoE, says James Nicoll, a neuropathologist at the UK's University of Southampton School of Medicine who has studied the protein's role in both diseases. For example, deposits of amyloid beta, the protein long fingered as a culprit in Alzheimer's pathology, are also present in many cases of CTE. "Another idea is that the repair process might work less well in people with $\varepsilon 4$," he says.

\section{By the numbers}

Despite the intrigue at a possible genetic cause behind CTE, researchers note that the data linking ApoE status to the disease are not wholly convincing. "A lot has been hung on $\varepsilon 4$ based on very small numbers," says Cantu. For example, a February study that examined the role of ApoE included only seven athletes with known ApoE genotypes (Neurosurgery doi:10.1227/NEU.0b013e318212bc7b, 2011).

In studies with tens of subjects, as most have been, "the effect is close to borderline statistical significance," says Nicoll. In a study of 89 subjects with head injury, his group found that ApoE $\varepsilon 4$ carriers were twice as likely as others to experience worse outcomes. But when the researchers repeated the study in more than 1,000 subjects, the ApoE $\varepsilon 4$ effect shrank dramatically (Lancet 350, 1069-1071, 1997; Brain 128, 2556-2661, 2005).

More data might be forthcoming from Cantu's institute, which houses a brain bank with some 70 CTE brains from athletes and military personnel. One of them belonged to Duerson, who, in his suicide note, charged his family to make sure his brain made its way to the institute. Not all the brains in the bank have been genotyped, says Cantu, declining to comment on Duerson's ApoE status. However, he says, "I must say we're not finding a high correlation" between CTE and the $\varepsilon 4$ variant.

A handful of other genetic elements have also been linked to CTE, but their role is largely speculative. These include the APOE promoter and the gene encoding catechol-Omethyltransferase, which is an enzyme that degrades dopamine and norepinephrine.

"There's really not a lot of evidence that we should be doing genetic testing before an athletic competition," says Vicki Kristman, an injury epidemiologist at the University of Toronto whose 2008 study of 318 athletes failed to find evidence that the $\varepsilon 4$ allele predisposed athletes to concussion (Clin. J. Sport. Med. 18, 322-328, 2008).

Even if ApoE $\varepsilon 4$ or other genetic variations could be scientifically validated as a screening tool for CTE susceptibility among athletes, serious questions would remain on how to implement it, says Jordan. One problem would be ensuring confidentiality, particularly because the test would also reveal a person's Alzheimer's risk. But on an even more basic level, says Jordan, it wouldn't be entirely clear what to do with the results. "Say someone is $\varepsilon 4$ positive. Can you stop them from competing?"

Alla Katsnelson 